\title{
Homoclinic bifurcations in Chua's circuit
}

\author{
Sandra Kahan*, Anibal C. Sicardi-Schifino \\ Instituto de Física, Universidad de la República, C.C. 30, C.P. 11 000, Montevideo, Uruguay
}

Received 1 April 1998; received in revised form 17 July 1998

\begin{abstract}
In this paper we study the possible relationship between the Birth of the Double Scroll [L.O. Chua et al., IEEE-CAS 33 (11) (1986) 1073] and the homoclinic bifurcations in the traditional Chua's equations. Using a one-dimensional Poincaré map we determine the existence of secondary symmetric homoclinic orbits of Shil'nikov type, born with the Chua's attractor, connecting unstable and stable manifolds of the trivial equilibrium point. In addition, taking into account the presence of other homoclinic orbits for the asymmetric attractor and heteroclinic orbits for the symmetric attractor (connecting unstable and stable manifold of the non-trivial equilibrium points), we suggest a hypothesis about the Birth of Double Scroll structure on the $(\alpha, \beta)$ plane. (c) 1999 Published by Elsevier Science B.V. All rights reserved.
\end{abstract}

PACS: $0.5 .45 .+\mathrm{b} ; 02.60 . \mathrm{Cb} ; 84.30$

Keywords: Homoclinic; Bifurcation; Electronic; Circuit

\section{Introduction}

Chua's circuit [1] has become a very interesting dynamical system, with a wide number of possible applications in electronics [2,3]. It is a rather simple electronic oscillator (in the simplest case, it consists of four linear elements and one nonlinear device) and its autonomous nature makes the system very suitable for laboratory and computer simulation studies. In this paper, we deal with the traditional Chua's equations, written in the following dimensionless form:

$$
\begin{aligned}
& \dot{x}=\alpha[y-h(x)], \\
& \dot{y}=x-y+z, \\
& \dot{z}=-\beta y, \\
& h(x)=b x+\frac{1}{2}(a-b)(|x+1|-|x-1|),
\end{aligned}
$$

*Corresponding author. Fax: +598-2-711-1630; e-mail: skahan@fing.edu.uy. 
where $\alpha$ and $\beta$ are positive real parameters and $h(x)$ is an odd-symmetric three segment piecewise-linear transference curve, with slopes $a$ and $b$.

System (1) presents three equilibrium points and the parameters $\alpha$ and $\beta$ do not affect their location. The planes $U_{ \pm 1}=\{(x, y, z) / x= \pm 1\}$, where $h(x)$ has discontinuous slopes define three different domains; we choose $a=-1 / 7$ and $b=2 / 7$ to locate the three equilibrium points at each region. Taking into account $k=(b-a) / b=3 / 2$ we have

$$
\begin{aligned}
& P_{+}=(k, 0,-k) \in D_{+1}=\{(x, y, z) / x>1\}, \\
& P_{0}=(0,0,0) \in D_{0}=\{(x, y, z) /|x| \leqslant 1\}, \\
& P_{-}=(-k, 0, k) \in D_{-1}=\{(x, y, z) / x<-1\} .
\end{aligned}
$$

In a wide zone of the parameter plane $(\alpha, \beta)$ the trivial equilibrium point $P_{0}$ is of saddle-focus type [4]. It has an unstable local manifold $E^{U}\left(P_{0}\right)$, given by a line and a stable local manifold $E^{S}\left(P_{0}\right)$, given by a plane. The non-trivial fixed points are of saddle-focus type too, but their stable local manifolds $E^{S}\left(P_{ \pm}\right)$are lines and their unstable local manifolds $E^{U}\left(P_{ \pm}\right)$are planes [1].

Although system (1) presents a great gallery of strange attractors [5], we focus our attention on the transition from two asymmetric attractors, called Rössler's attractors [6], to a symmetric attractor, called Chua's attractor or Double Scroll [7]. Rössler's attractor is characterized by a flow around the trivial and one of the non-trivial equilibrium points, while the flow in Chua's attractor involves the stable and unstable local manifolds of the three equilibrium points. Experimentally this transition, called the Birth of the Double Scroll, was described by Matsumoto et al. [7] like an abrupt glue of the asymmetric chaotic attractors. This author also described the Death of the Double Scroll, by a collision of the attractor with a saddle-type close orbit, which surrounded the Double Scroll.

Chua's system (1) also presents a symmetric principal homoclinic orbit of Shil'nikov type, around the trivial equilibrium point, which can be numerically observed after the Death of the Double Scroll has taken place. This has been shown for a system with a cubic polynomial odd-symmetric transference curve, by Khibnik et al. [4].

In Section 2 we define a one-dimensional map for system (1) to be used in the analysis of the global unstable structure of the homoclinic phenomena. We also describe how the behavior of this map becomes complex, when we approach the Birth of the Double Scroll frontier, when we increase the $\alpha$ value, from the critical value of a spiral-screw Rössler's transition.

In Section 3 we calculate several periodic orbits which can be found in the Rössler's and Chua's attractors and show how they lead to secondary homoclinic orbits of Shil'nikov type [8], connecting unstable and stable manifolds of the trivial equilibrium point.

In Section 4 we sketch the several changes in the dynamical behavior of the attractors on the parameters plane and how they are related with homoclinic systems for the 


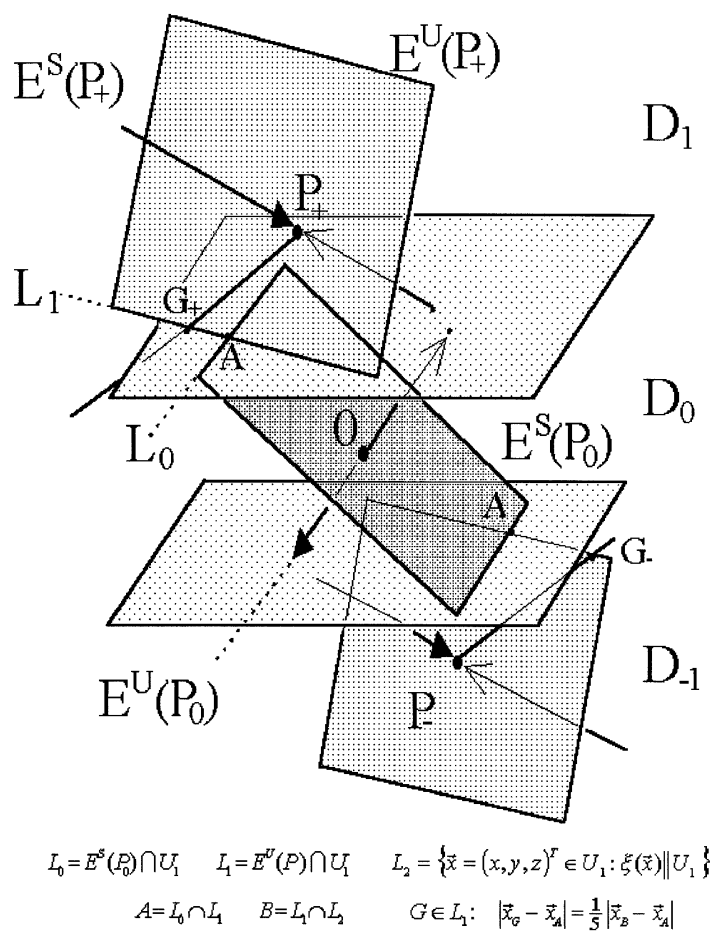

Fig. 1. Eigenspaces of the equilibria. $\xi(\overrightarrow{\boldsymbol{r}}) \| U_{1}$ means the vector field lies on a plane parallel to $U_{1}$.

asymmetric attractor and heteroclinic systems for the symmetric attractor, connecting the unstable and stable manifolds of the non-trivial equilibrium points.

Finally, in Section 5, taking into account the global behavior of those homoclinic and heteroclinic systems, we suggest a hypothesis about the Birth of the Double Scroll structure on the $(\alpha, \beta)$ plane.

\section{One-dimensional Poincaré map}

In a wide zone of the parameters plane $(\alpha, \beta)$ the Jacobian matrix, evaluated at the non-trivial equilibrium points $P_{ \pm}$, has a real negative eigenvalue whose modulus is large compared with the other eigenvalues [1]. Then, we assume that trajectories, coming from the $D_{0}$ region to the $D_{ \pm 1}$ regions are attracted almost instantaneously to the unstable manifolds $E^{U}\left(P_{ \pm}\right)$.

This property allows us to define a one-dimensional map (1-D map), computing the flow transition across two semi-infinite planes: $W_{+}$and $W_{-}$, perpendicular to $E^{U}\left(P_{+}\right)$ and $E^{U}\left(P_{-}\right)$, respectively, and containing the non-trivial equilibrium points. The flow strikes $W_{ \pm}$near the planes $E^{U}\left(P_{ \pm}\right)$. To improve this behavior, we choose points $G_{ \pm}$on lines $L_{ \pm 1}=E^{U}\left(P_{ \pm}\right) \cap U_{ \pm}$near the points $A_{ \pm}=E^{S}\left(P_{0}\right) \cap U_{ \pm}$, as shown in Fig. 1 . The definition of $W_{ \pm}$(not shown in Fig. 1) is completed taking into account the condition 
$P_{ \pm} G_{ \pm}=E^{U}\left(P_{ \pm}\right) \cap W_{ \pm}$which corresponds to the semi-infinite lines where the flow approximately crosses $W_{ \pm}$. We take like a measure of the cross point position $(r)$, the quadratic distance from this point to $P_{+}$or $P_{-}$, according to the plane $\left(W_{+}\right.$or $W_{-}$, respectively) to which the cross point belongs. Although we have two different semi-infinite planes (one in each subspace defined by the stable manifold of the trivial equilibrium point $E^{S}\left(P_{0}\right)$ ), we compute only one 1D-map [9]

$$
f: W_{+} \cup W_{-} \rightarrow W_{+} \cup W_{-} / r_{n+1}=f\left(r_{n}\right) .
$$

For Rössler's attractor, the flow that begins at the upper subspace, remains in this subspace. Then, a cross point and its first iterate are computed on $W_{+}$. The $r_{n}$ and $r_{n+1}$ coordinates of the 1-D map are the quadratic distance from the cross points to $P_{+}$. For Chua's attractor, the flow that begins at the upper subspace can travel to the lower subspace. In this case, we compute a cross point on $W_{+}$and its first iterate on $W_{-}$. The $r_{n}$ abscissa of the 1-D map is the quadratic distance from the cross point to $P_{+}$ and the $r_{n+1}$ ordinate is the quadratic distance from this point to $P_{-}$. The following iterate can be computed on $W_{-}$or $W_{+}$, depending on whether the flow remains on the lower subspace or travels to the upper subspace.

Additionally, for Rössler's attractor, we define the points $H_{ \pm}$(or simply point $H$ ) on the $P_{ \pm} G_{ \pm}$(or simply $P G$ semi-infinite line) as the point of maximum abscissa in the 1-D map. For Chua's attractor, we define $H$ as the point on the $P G$ line for which the flow strikes exactly on the trivial equilibrium point stable manifold $E^{S}\left(P_{0}\right)$. Then, in both cases, we normalize the 1-D map coordinate by taking the quadratic distance of the point $H$ to the equilibrium point $P$ as $r=1$.

Fig. 2 shows the 1-D map (3) for $\alpha$ and $\beta$ values for which the Double Scroll attractor is observed. We remark the presence of small oscillations at both sides of the $H$ point position on the map, which represent the winding flow around the unstable manifold $E^{U}\left(P_{0}\right)$, in the $D_{0}$ region and near the stable manifold $E^{S}\left(P_{0}\right)$. By fixing $\beta=14.3$ and increasing $\alpha$ continuously, we observed an increase in the number of relative extremum. The asymmetric ones (left $H$ ) in Rössler's attractor are observed for $8.80<\alpha<8.813232$ and the symmetric ones (right $H$ ) in Chua's attractor for $8.813232<\alpha<10.73$. The parameter's value estimated for the Birth of the Double Scroll is $\alpha_{B D S}=8.813232$ and $\alpha_{D D S}=10.73$ is the parameter value estimated for the Death of the Double Scroll for which the dynamics has been completed. For $\alpha_{B D S}$ we observe several of these small oscillations at the left side of $H$, by increasing the accuracy of the calculation. We believe that there would be an infinite number of oscillations if the exact $\alpha_{B D S}$ could be numerically achieved [8]. For $\alpha=8.815$ we also observe several of those oscillations close to and on the right side of point $H$. Chua's attractor has been born in a complex manner.

\section{Secondary homoclinic orbits}

The spiral-screw chaos transition line $\Lambda_{a}$ in the two parameter plane (see Fig. 3) is characterized by the rising of the right-hand branch of the first relative minimum 

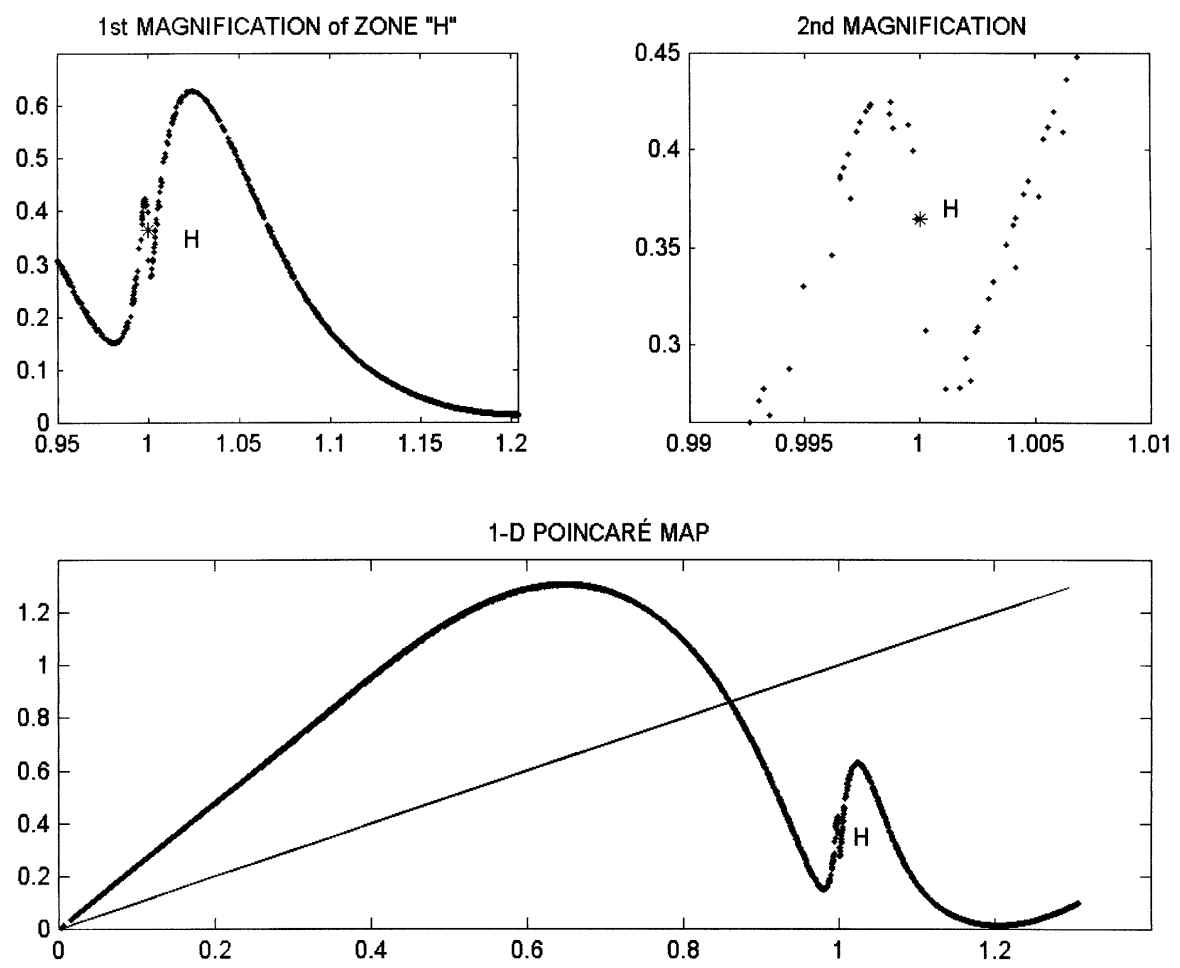

Fig. 2. 1-D map for $\alpha=9.375$ and $\beta=14.3$. The figures at the top show several oscillations around the point $H$ of abscissa $r_{n}=1$.

at the 1-D map [11]. Gaspard et al. [10] pointed out that there are two families of superstable period- $n$ orbits constructed by requiring that the maximum or minimum of the Rössler's 1-D map belongs to the orbit. These two families of superstable orbits with increasing periods accumulate on the end point of a line of a homoclinic system $\Gamma_{a}$ in a two parameter plane given by the coordinates of the minimum.

Taking the 1-D map described in the last section, for a mesh of the parameter values $(\alpha, \beta)$ we interpolate numerically the shape of the map in the linear and quadratic principal maximum regions. We also estimate the relative extrema coordinates and the point $H$ ordinate, for a wide zone of the $(\alpha, \beta)$ plane. Then, we calculate the geometrical locus of the period-3 orbits spawned by the relative extrema of the 1-D map. Observing the principal maximum behavior on the 3-map (defined as: $r_{n+3}=f^{3}\left(r_{n}\right)$ ), we sketch the period-3 geometrical locus on the parameters plane spawned by this maximum, as Fig. 3 shows. Period-3 orbits with an iterate at one of the relative extremum have two branches: the lower (upper) branch which corresponds to an orbit that starts at the relative extremum, has one iterate at the linear region of the 1-D map and one iterate at the quadratic maximum region, left- (right-) hand side of this maximum. Each branch is born at a new transition chaos-chaos $\Lambda$ line in the $(\alpha, \beta)$ plane. The birth point corresponds to a doubly superstable period-3 orbit [10]. We also remark 


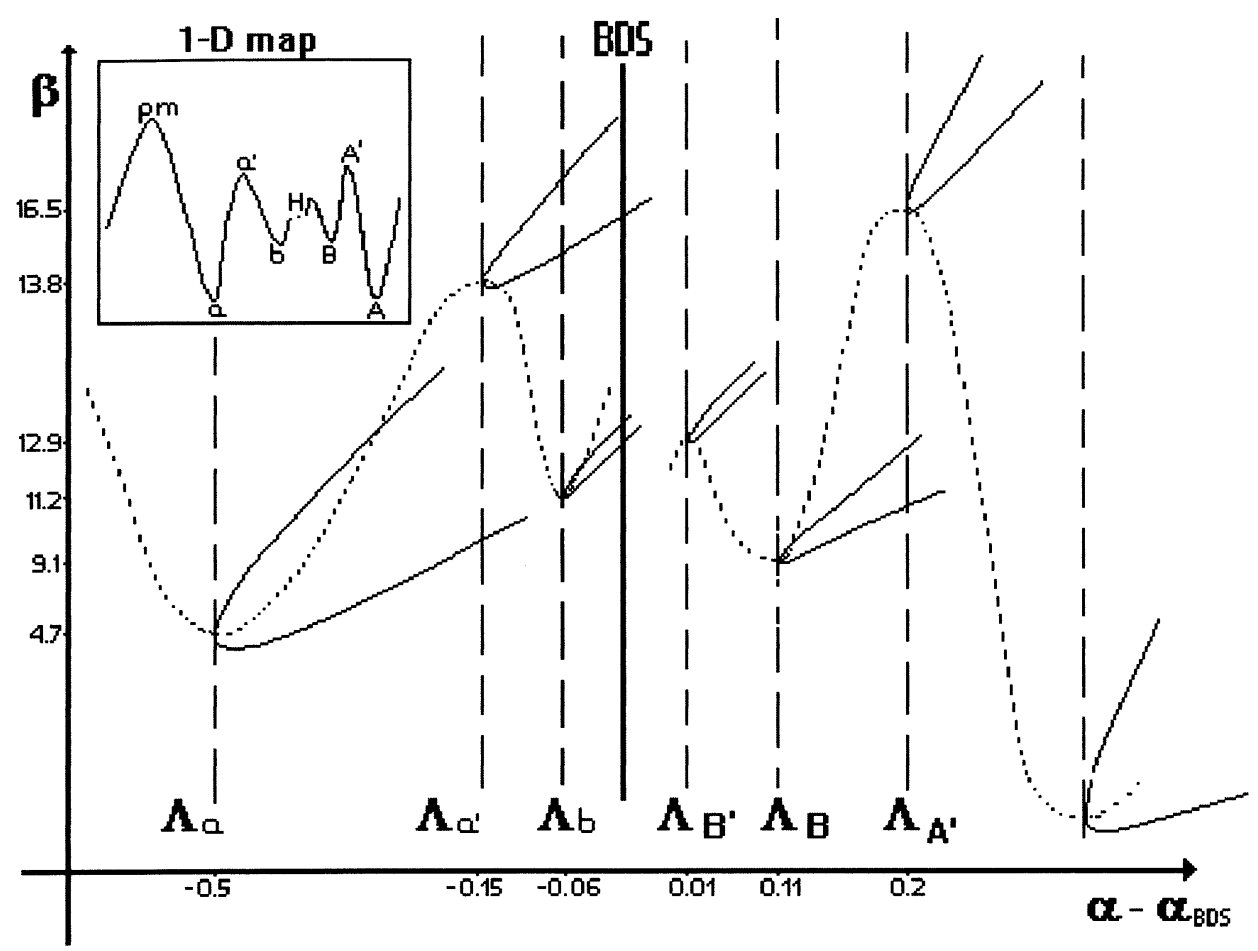

Fig. 3. Period-3 orbits spawned by the relative extrema (solid lines) and by the principal maximum (dotted line) in $(\alpha, \beta)$ plane. The BDS line marks the Rössler-Chua transition. The dashed lines show the several dynamical changes in Rössler's attractor (left side BDS) and Chua's attractor (right side BDS). The top-left figure shows a scheme of the 1-D map: pm-principal maximum; lower case and capital letters name the relative extrema around the point $H$.

that the period-3 orbits to which the principal maximum belongs are continuous in the parameter plane.

We focus on the period-3 orbits spawned by point $H$, for which the symmetrical flow winds three times around one of the non-trivial equilibrium points and injects exactly at the trivial equilibrium point stable manifold (3-H orbits) [12]. Moreover, we estimate the geometrical locus in $(\alpha, \beta)$ of a sequence of period- $n$ orbits $(n=2,3,4)$ that consist of $(n-2)$ iterates on the linear region of the map and one iterate on the quadratic maximum region (left and right to this maximum), starting at point $H$. Because of the definition of this point, these period- $n$ orbits are secondary symmetrical homoclinic orbits of Shil'nikov type [8] connecting the unstable and stable $P_{0}$ manifolds, winding $n(n=2,3,4)$ times the non-trivial equilibrium points. Fig. 4 shows its geometrical locus in $(\alpha, \beta)$ plane. Indeed, each $n-H$ curves has two branches: $n l-H$ (upper branch) and $n r-H$ (lower branch).

From the analysis of the $n$-map, near the point $H$, we observe asymmetric and symmetric period- $n$ orbits for parameters values near $(\alpha, \beta)$ values for which a secondary homoclinic orbit is observed. Fixing the value of $\beta$, varying $\alpha$ continuously and taking into account the time that the period- $n$ orbit takes to complete a cycle, we can group 

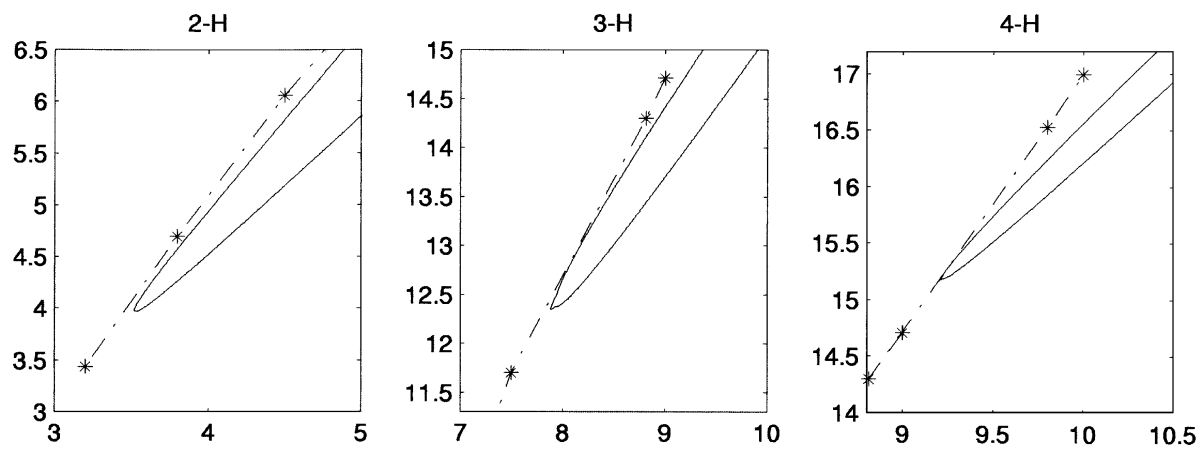

SECONDARY HOMOCLINIC ORBITS

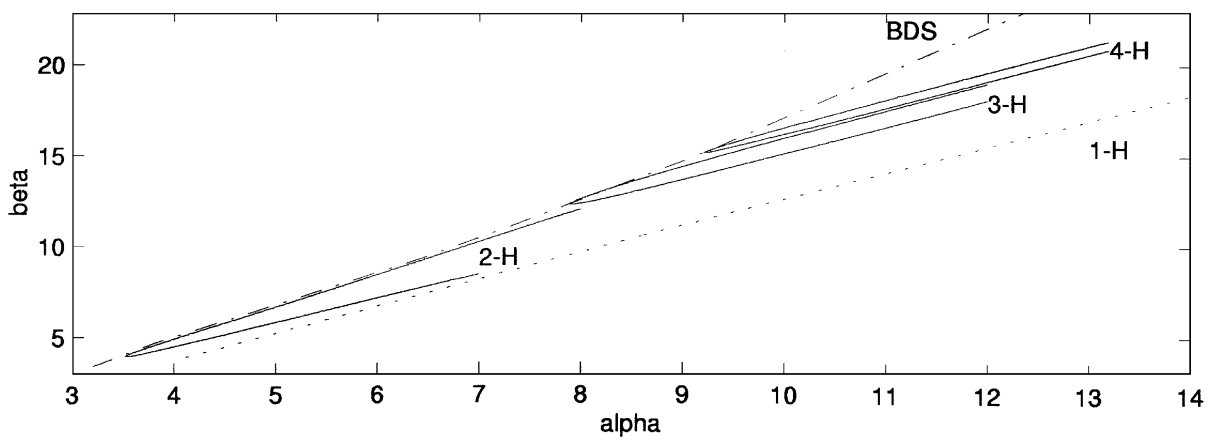

Fig. 4. Geometrical locus in $(\alpha, \beta)$ of secondary homoclinic orbits $(2-H),(3-H)$ and $(4-H)$ (solid lines). The dashed line (1-H) shows the principal homoclinic orbit around $P_{0}$. The dashed-dotted line (BDS) shows the Birth of Double Scroll attractor. Top figures show the merging points of the $n-H$ branches near the BDS line. Asterisks mark computational data.

them in wiggles around the $\alpha_{n-H}$ value (for which a $n l-H$ or a $n r-H$ homoclinic orbit is observed). From the local analysis developed by several authors $[8,10]$, we know that the period- $n$ orbits are of saddle-stability type for $\alpha$ values near $\alpha_{n-H}$. They have small windows (in the parameter $\alpha$ ) of defined stability: for system (1) these windows are stable if $\alpha<7$ and totally unstable if $\alpha>7$. These windows can be observed near parameter values where a tangent bifurcation occurs. Thus, the continuous branches of period-3 orbits spawned by the relative extremum of the map (see Fig. 3) show approximately the locus in the $(\alpha, \beta)$ plane of these totally defined stability windows.

\section{Homoclinic and heteroclinic orbits}

For system (1) the end point of the homoclinic system $\Gamma_{a}$ associated with the spiral-screw transition $\Lambda_{a}$ [10] is observed at $\alpha=14.7$ and $\beta=29.0$. These homoclinic orbits are asymmetric and connect the unstable and stable manifolds of the non-trivial equilibrium point. The minimum associated to this first transition has a null 


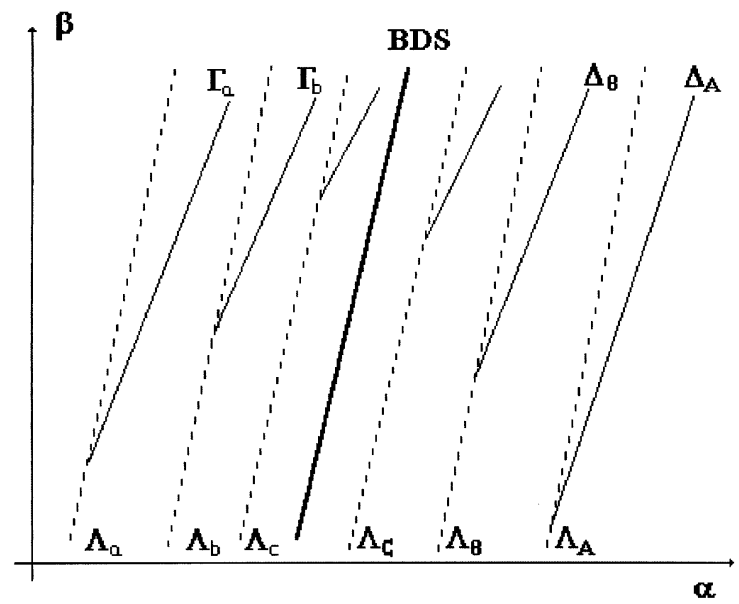

Fig. 5. Dynamical changes in Rössler's and Chua's attractors (dashed lines) and their homoclinic $\Gamma$ and heteroclinic $\Delta$ systems (solid lines) in the parameters' plane. The BDS line denotes the Birth of the Double Scroll attractor.

ordinate in the 1-D map. In Section 2 we have pointed out that the number of relative minima increases for parameters values between this first chaos-chaos transition and the Birth of Double Scroll. Each new dynamical transition on the Rössler's attractor is associated with the presence of a new homoclinic system [11] (Fig. 5). On the other hand, when Chua's attractor is observed, the number of relative minima at the right of point $H$ increases when we increase the $\alpha$ value, fixing the $\beta$ value. Then, we point out the presence of a line of heteroclinic systems $\Delta$ whose end point is on the respective transition lines on the $(\alpha, \beta)$ plane (Fig. 5). In particular, for $\alpha=9.2$ and $\beta=14.3$ we observe a 1-D map with a rising most right minimum with null ordinate: the unstable $P_{+}$manifold is connected with stable $P_{-}$manifold and unstable $P_{-}$manifold is connected with the $P_{+}$stable manifold, creating the $\Delta_{A}$ heteroclinic line on the $(\alpha, \beta)$ plane.

\section{Conclusions}

We find three families of secondary homoclinic orbits merging with Chua's attractor. Each branch of the $n-H$ homoclinic family is associated with two wiggles of period- $n$ orbits: one for the asymmetric and one for the symmetric cycles. Windows (in the parameters plane) characterized by a defined stability may explain the sequence of alternating chaotic and periodic behavior of system (1) reported by several authors [1] and [7].

We also have numerical evidence that there are other secondary homoclinic orbits of Shil'nikov type of higher order and we suppose that they will accumulate at some point on the BDS line. 
We also point out several chaos-chaos transitions in the sinus of Rössler's (asymmetric) attractor in the $(\alpha, \beta)$ plane. Each transition is associated with the end point of a line $\Gamma$ that represents a family of homoclinic orbits around the non-trivial equilibrium point which accumulate on the Birth of the Double Scroll line by the left. Chua's (symmetric) attractor also exhibits several chaos-chaos transitions on the parameter plane, which are associated with heteroclinic closed orbits between the non-trivial equilibrium points which accumulate on the Birth of Double Scroll line by the right.

Then, there would exist an accumulating line of heteroclinic orbits with an end point on the Birth of Double Scroll frontier. This heteroclinic family would be present for a set of $(\alpha, \beta)$ values for which a symmetric closed orbit exists. This orbit must connect the stable $P_{0}$ manifold with both unstable $P_{ \pm}$manifolds, and both stable $P_{ \pm}$manifolds with the unstable $P_{0}$ manifold.

In brief, reporting the complex homoclinic structure on the parameters plane of the traditional Chua's equations, we try to clarify the bifurcation phenomena associated with the Birth of the Double Scroll attractor. Furthermore, this structure provides a plausible interpretation of a number of experimental results reported in electronics applications $[2,13,14]$ for which alternating periodic and chaotic sequences are observed.

\section{References}

[1] L.O. Chua, M. Komuro, T. Matsumoto, IEEE-CAS 33 (11) (1986) 1073.

[2] H. Dedieu, M.P. Kennedy, M. Hasler, IEEE-CAS 40 (10) (1993) 634.

[3] L. Kocarev, K. Eckart, L.O. Chua, U. Parlitz, Int. J. Bifurcation Chaos 2 (1992) 705.

[4] A.I. Khibnik, D. Roose, L.O. Chua, Int. J. Bifurcation Chaos 3 (2) (1993) 363.

[5] L.P. Shil'nikov, Int. J. Bifurcation Chaos 4 (3) (1994) 489.

[6] O.E. Rössler, Ann. N. Y. Aca. Sci. 31 (1979) 376.

[7] T. Matsumoto, L.O. Chua, Physica D 24 (1987) 97.

[8] P. Glendinning, C. Sparrow, J. Stat. Phys. 35 (5,6) (1984) 645.

[9] M.J. Pacifico, A. Rovella, M. Viana, IMPA-Preprints, A-136, 1996.

[10] P. Gaspard, R. Kapral, G. Nicolis, J. Stat. Phys. 35 (5,6) (1984) 697.

[11] P. Gaspard, G. Nicolis, J. Stat. Phys. 31 (3) (1983) 499.

[12] E. Freire, A.J. Rodriguez-Luis, E. Gamero, E. Ponce, Physica D 62 (1993) 230.

[13] J.M. Cruz, L.O. Chua, IEEE-CAS 40 (10) (1993) 614.

[14] G. Mayer-Kreiss, I. Choi, N. Weber, R. Bargar, A. Hübler, IEEE-CAS 40 (10) (1993) 688. 